\title{
Santé et sécurité du travail au Saguenay- Lac-Saint-Jean : pour un regard exhaustif
}

\author{
Cheikh Faye ${ }^{a}$, Ibrahima Pouye ${ }^{b}$
}

RÉSUMÉ. Il existe plusieurs données relatives à la région du Saguenay-Lac-Saint-Jean dans le domaine de la santé et sécurité au travail (SST). Cependant, elles sont éparses et parfois incomplètes. Elles ne permettent pas de poser un regard global et exhaustif sur l'état de la situation ni de renseigner, avec précision, sur l'ampleur des principales problématiques et leurs interactions. L'objectif principal du présent article est de dresser un portrait de la région dans le domaine de la SST. La recherche réalisée est de nature exploratoire et descriptive. Elle a donné lieu à l'analyse secondaire de données publiées par des institutions nationales et des organismes régionaux.

\begin{abstract}
There are several data relating to the Saguenay-Lac-Saint-Jean region in the field of occupational health and safety (OHS). But they are scattered and sometimes incomplete. They do not provide a global and exhaustive view of the situation nor do they provide precise information on the extent of the main issues and their interactions. The main objective of this article is to provide a picture of the region in the field of OSH. The research carried out is exploratory and descriptive in nature. It entailed the analysis of data already published by national institutions and regional bodies, in order to understand and describe the regional situation in the field of OSH.
\end{abstract}

\section{Introduction}

La recherche en santé et sécurité au travail (SST) comme processus de production de connaissances pratiques, transférables et utiles aux organisations nécessite, au préalable, de dresser un portrait de la situation et d'identifier des besoins auxquels les milieux de travail sont confrontés.

Les statistiques publiées par les institutions nationales compétentes et les organismes régionaux intéressés ou évoluant dans le domaine de la SST concernent, pour l'essentiel, des données rétrospectives. En effet, les statistiques disponibles permettent d'esquisser un fidèle portrait des lésions professionnelles (accidents du travail et maladies professionnelles) déclarées et acceptées. Elles renseignent sur des faits qui sont déjà survenus. Si elles fournissent de précieuses informations, elles demeurent insuffisantes pour offrir une vision globale et exhaustive permettant, par exemple, d'identifier des risques potentiels ou de nouveaux risques, en vue de cibler ou de développer des actions de prévention adéquates.

Les mutations technologiques, organisationnelles et économiques font émerger de nouveaux risques, de nouvelles problématiques. Ainsi, de nouvelles formes de gestion ou d'initiatives développées par les milieux de travail pour y faire face ne ressortent pas au sein des statistiques généralement publiées. Par conséquent, pour identifier des axes de recherche novateurs et cibler des activités de prévention pertinentes, il faut une meilleure connaissance de la situation en SST, en particulier dans la région du SaguenayLac-St-Jean, qui constitue notre premier territoire d'intervention. D'où la pertinence de cette recherche dont le but ultime est de combler ce besoin. En effet, une telle recherche se justifie, car, autant que nous sachions, il n'existe pas encore de portait en SST qui, entre autres, décrirait et expliquerait les principales caractéristiques de la région en la matière.

\footnotetext{
a Professeur, Université du Québec à Chicoutimi

b Doctorant, Université du Québec à Chicoutimi 
Les résultats présentés dans le présent article se limitent à ceux obtenus au terme de la première phase de notre recherche, c'est-à-dire au recueil et à l'analyse des principales données permettant de décrire le portrait de la région en matière de SST. Les résultats de la seconde phase (réalisation d'une étude qualitative en vue d'identifier les priorités régionales en SST) feront l'objet d'une publication ultérieure.

\section{Méthodologie}

Une recherche peut viser "la découverte ou l'exploration lorsque le phénomène à l'étude est peu connu, soit que les connaissances que l'on possède sont rares ou inexistantes, soit que le phénomène est encore mal élucidé » (Fortin et Gagnon, 2016, 16). Pour Mayer (2000), une analyse du milieu permet de déterminer les principales caractéristiques de celui-ci et, subséquemment, d'orienter l'action. D’où la nécessité et la pertinence de réaliser une étude, à la fois exploratoire et descriptive, visant, d'une part, à dresser un portrait de la région dans le domaine de la SST et, d'autre part, à identifier les principales problématiques considérées comme prioritaires.

La collecte des données s'est déroulée en deux phases, correspondant à un des deux objectifs de la recherche. Pour la phase 1 (objectif 1), nous avons utilisé des méthodes quantitatives en colligeant les principaux agrégats pertinents et statistiques concernant la région dans le domaine de la SST. Concernant la phase 2 (objectif 2), nous ferons appel à des méthodes qualitatives (sondages, entrevues et observations directes) afin d'identifier les priorités en matière de recherche et d'action.

Dans la phase 1, les données collectées et analysées sont des données secondaires, c'est-à-dire des "éléments informatifs tirés d'un regroupement de données primaires et rassemblés pour des fins autres que celles pour lesquelles ces données ont été recueillies initialement» (Bernatchez et Turgeon, 2016, p. 504). C'est ainsi que les données pertinentes publiées par les principaux organismes gouvernementaux (MSSS, CNESST, INSPQ, ISQ, etc.) ${ }^{1}$ et régionaux (CLD, CIUSSS, etc.) $)^{2}$ sont collectées et analysées. Le recours aux données secondaires permet, entre autres, de "retourner dans le passé et d'analyser le changement à partir d'indicateurs prélevés en temps réel [...] » (Bernatchez et Turgeon, 2016, p. 507).
Les sources des données secondaires colligées et analysées sont multiples et variées : rapports sur les statistiques annuelles, rapports pluriannuels concernant certaines thématiques de la SST (surdité, violence au travail, stress en milieu de travail, etc.), résultats d'enquêtes sur la population active, portraits socio-économiques régionaux, portraits de santé régionaux, répertoires des entreprises, etc. Toutes ces sources sont directement accessibles par Internet et sont éditées sur des supports téléchargeables.

\section{Résultats}

La présentation des résultats de la phase 1 de cette recherche s'articule principalement autour de trois points : une description du portrait général démographique et économique de la région pour planter le décor (2.1); une esquisse du portrait régional des lésions et risques professionnels (2.2); une présentation de certaines initiatives de prévention dans la région (2.3).

\subsection{Portrait général démographique et économique}

Au 1 er juillet 2016, la région administrative du Saguenay-Lac-Saint-Jean comptait 277232 habitants sur les 8326089 recensés au Québec à la même date (ISQ, 2017a). Ce qui la place, en ordre d'importance, au dixième rang des 17 régions administratives qui composent la province. Le groupe d'âge 20-64 ans représente 59,8\% de la population régionale, soit 165679 personnes (ISQ, 2017a). En juillet 2017, le nombre d'emplois est estimé à 128800 (ISQ, 2017b). Selon le régime de travail, 21,4\% des emplois de la région sont à temps partiel et 78,6\% sont des emplois permanents (ISQ, 2017a).

Le taux de couverture syndicale s'établit à 45,9\% plaçant ainsi la région du Saguenay-Lac-SaintJean en quatrième position à l'échelle provinciale tout juste derrière les régions de l'Outaouais, de la Côte-Nord et du Nord-du-Québec (CloutierVilleneuve et Demers, 2014). La région affiche un niveau de syndicalisation supérieur à celui du Québec (39,6 \%) devant des régions comme celles de Québec, de Laval et de Montréal.

Selon les données désaisonnalisées de juillet 2017, le taux de chômage dans la région s'établit à $7,5 \%$, soit une baisse de 0,3 point en six mois 
(ISQ, 2017b). Le Saguenay-Lac-Saint-Jean a ainsi un taux de chômage plus important que celui de l'ensemble de la province $(5,9 \%)$ et ne surpasse que 3 régions administratives sur les 17 que compte le Québec.

La CNESST (2016) fait état, en 2015, de 11202 établissements actifs ${ }^{3}$ dans la région. Comme le montre le tableau 1, plus de $53 \%$ des entreprises répertoriées dans la région sont très petites, occupant quatre salariés ou moins (CLD, 2017). Les entreprises comptant moins de 50 salariés représentent $95,2 \%$, soit la quasi-totalité des entités répertoriées dans la région. Seulement, 4 entreprises embauchent plus de 1000 travailleurs.

\begin{tabular}{|l|c|}
\hline \multicolumn{1}{|c|}{ Nombre d'emplois } & \multicolumn{1}{|c|}{$\begin{array}{c}\text { Répartition des entreprises } \\
\text { selon les effectifs (en \%) }\end{array}$} \\
\hline 1 à 4 & 53,1 \\
\hline 5 à 19 & 32,4 \\
\hline 20 à 49 & 9,7 \\
\hline 50 à 99 & 3,3 \\
\hline 100 à 199 & 1 \\
\hline 200 à 499 & 0,4 \\
\hline 500 à 999 & 0,1 \\
\hline 1000 et plus & 0 \\
\hline Total & 100 \\
\hline
\end{tabular}

Tableau 1 - Nombre d'entreprises et leur répartition selon les effectifs Source : Centre local de développement (CLD) Maria-Chapdelaine (2017).

La CNESST classe les employeurs couverts par le régime québécois de SST selon la nature de leurs activités principales dans 32 secteurs d'activité économique (SAE). Ces derniers sont regroupés en six groupes selon la fréquence et la gravité des accidents du travail et maladies professionnelles qui y ont été enregistrés (groupes prioritaires). Les employeurs classés dans le groupe 1 exercent des activités plus dangereuses (en matière de fréquence et de gravité des lésions professionnelles) que ceux du groupe 6, par exemple. Une compilation de la liste des employeurs classifiés par groupe prioritaire (1 à 6 ) pour la région du Saguenay-Lac-Saint-Jean établie par la CNESST (2017a) permet d'avoir une idée sur la ventilation des entreprises présentes dans la région selon le groupe prioritaire et le SAE. En effet, près de $70 \%$ des entreprises évo- luant dans la région relèvent des groupes prioritaires 3, 4 et 6 tandis que le reste est réparti dans les groupes prioritaires 1, 2 et 3 (tableau 2). Dans le premier lot, soit $70 \%$ des entreprises, on note la présence, largement dominante, d'entreprises relevant des secteurs du " commerce » (SAE 16), des «autres services commerciaux et personnels » (SAE 21), des " services médicaux et sociaux » (SAE 30), de l'« agriculture » (SAE 26), des "finances, assurances et affaires immobilières » (SAE 29) et de l'« enseignement et services annexes »(SAE 28). Le second lot, soit les $30 \%$ restants (groupes prioritaire 1 à 3), est composé, en grande majorité, d'entreprises des secteurs " bâtiment et travaux publics » (SAE 1), du " transport et entreposage » (SAE 15), de la «forêt et scieries » (SAE 3), de la « fabrication de produits en métal » (SAE 5) et de l'« administration publique ». 


\begin{tabular}{|c|c|c|}
\hline Groupes prioritaires & Secteurs d'activité économique & $\%$ \\
\hline Groupe prioritaire 1 & $\begin{array}{l}\text { 1. Bâtiment et travaux publics; } \\
\text { 2. Industrie chimique; } \\
\text { 3. Forêt et scieries; } \\
\text { 4. Mines, carrières et puits de pétrole; } \\
\text { 5. Fabrication de produits en métal. }\end{array}$ & 18,8 \\
\hline Groupe prioritaire 2 & $\begin{array}{l}\text { 6. Industrie du bois (sans scierie); } \\
\text { 7. Industrie du caoutchouc et des produits en matière plastique; } \\
\text { 8. Fabrication d'équipement de transport; } \\
\text { 9. Première transformation des métaux; } \\
\text { 10. Fabrication des produits minéraux non métalliques. }\end{array}$ & 1,8 \\
\hline Groupe prioritaire 3 & $\begin{array}{l}\text { 11. Administration publique; } \\
\text { 12. Industrie des aliments et boissons; } \\
\text { 13. Industrie du meuble et des articles d'ameublement; } \\
\text { 14. Industrie du papier et des activités diverses; } \\
\text { 15. Transport et entreposage. }\end{array}$ & 9,9 \\
\hline Groupe prioritaire 4 & $\begin{array}{l}\text { 16. Commerce; } \\
\text { 17. Industrie du cuir; } \\
\text { 18. Fabrication de machines (sauf électriques); } \\
\text { 19. Industrie du tabac; } \\
\text { 20. Industrie du textile. }\end{array}$ & 17,9 \\
\hline Groupe prioritaire 5 & $\begin{array}{l}\text { 21. Autres services commerciaux et personnels; } \\
\text { 22. Communications, transport d'énergie et autres services publics; } \\
\text { 23. Imprimerie, édition et activités annexes; } \\
\text { 24. Fabrication de produits du pétrole et du charbon; } \\
\text { 25. Fabrication de produits électriques. }\end{array}$ & 29,8 \\
\hline Groupe prioritaire 6 & $\begin{array}{l}\text { 26. Agriculture; } \\
\text { 27. Bonneterie et habillement; } \\
\text { 28. Enseignement et services annexes; } \\
\text { 29. Finances, assurances et affaires immobilières; } \\
\text { 30. Services médicaux et sociaux; } \\
\text { 31. Chasse et pêche; } \\
\text { 32. Industries manufacturières diverses. }\end{array}$ & 21,9 \\
\hline
\end{tabular}

Tableau 2 - Répartition des employeurs de la région selon le groupe prioritaire (1 à 6) et le secteur d'activité économique (SAE) Source : CNESST (2017), Liste des employeurs classifiés par groupe prioritaire (1 à 6) pour la région du Saguenay-Lac-Saint-Jean. Adaptée par les auteurs.

\subsection{Portrait régional des lésions et risques professionnels}

Entre 2012 et 2015, le nombre de dossiers de lésions professionnelles (accidents du travail et maladies professionnelles) ouverts et acceptés 4 par la CNESST concernant les travailleurs de la région est passé de 3282 dossiers en 2012 à 2943 dossiers en 2015 (tableau 3). Le nombre de dossiers pour lésions professionnelles ouverts et acceptés concernant les travailleurs de la région représente $4 \%$ du total enregistré pour l'ensemble du Québec au cours de la période considérée. Cette légère baisse masque une disparité dans l'évolution des lésions professionnelles selon la catégorie. En effet, si la tendance des accidents du travail est à la baisse, ce n'est pas le cas pour les maladies professionnelles qui sont passées de 265 en 2012 à 626 en 2015, soit une augmentation de plus de $136 \%$ en quatre années. De plus, il convient d'ajouter que la baisse des accidents du travail n'est pas spécifique à la région, car on constate une tendance similaire à l'échelle de la province. 


\begin{tabular}{|c|c|c|c|c|c|c|c|c|}
\hline & \multicolumn{2}{|l|}{2012} & \multicolumn{2}{|c|}{2013} & \multicolumn{2}{|c|}{2014} & \multicolumn{2}{|c|}{2015} \\
\hline & Nombre & $\%$ & Nombre & $\%$ & Nombre & $\%$ & Nombre & $\%$ \\
\hline $\begin{array}{l}\text { Nombre d'accidents du travail } \\
\text { Saguenay-Lac-St-Jean }\end{array}$ & 3282 & 3,8 & 3234 & 3,9 & 2955 & 3,6 & 2943 & 3,6 \\
\hline Province de Québec & 85523 & & 83458 & & 82321 & & 81765 & \\
\hline $\begin{array}{l}\text { Nombre de maladies professionnelles } \\
\text { Saguenay-Lac-St-Jean }\end{array}$ & 265 & 6,4 & 189 & 3,8 & 503 & 8,8 & 626 & 10,7 \\
\hline Province de Québec & 4117 & & 4910 & & 5725 & & 5853 & \\
\hline $\begin{array}{l}\text { Total } \\
\text { Saguenay-Lac-St-Jean }\end{array}$ & 3547 & 4 & 3423 & 3,9 & 3458 & 3,9 & 3569 & 4,1 \\
\hline Province de Québec & 89640 & & 88368 & & 88046 & & 87618 & \\
\hline
\end{tabular}

Tableau 3-Nombre de dossiers pour lésions professionnelles ouverts et acceptés Source : Statistiques annuelles de 2012, 2013, 2014 et 2015 de la CSST.

Les lésions professionnelles enregistrées au Saguenay-Lac-Saint-Jean ont entraîné le décès de 51 travailleurs entre 2012 et 2015 (tableau 4). Plus de
$60 \%$ de ces décès sont survenus à la suite d'une maladie professionnelle.

\begin{tabular}{|c|c|c|c|c|c|c|c|c|}
\hline & \multicolumn{2}{|c|}{2012} & \multicolumn{2}{|c|}{2013} & \multicolumn{2}{|c|}{2014} & \multicolumn{2}{|c|}{2015} \\
\hline & Nombre & $\%$ & Nombre & $\%$ & Nombre & $\%$ & Nombre & $\%$ \\
\hline $\begin{array}{l}\text { Décès à la suite d'un accident du travail } \\
\text { Saguenay-Lac-St-Jean }\end{array}$ & 8 & 10,7 & 4 & 6,3 & 4 & 7 & 4 & 5,8 \\
\hline Province de Québec & 75 & & 63 & & 57 & & 69 & \\
\hline $\begin{array}{l}\text { Décès à la suite d'une maladie professionnelle } \\
\text { Saguenay-Lac-St-Jean }\end{array}$ & 10 & 7,4 & 6 & 5 & 11 & $\begin{array}{c}10, \\
3\end{array}$ & 4 & 3,1 \\
\hline Province de Québec & 136 & & 121 & & 107 & & 127 & \\
\hline $\begin{array}{l}\text { Total } \\
\qquad \text { Saguenay-Lac-St-Jean }\end{array}$ & 18 & 8,5 & 10 & 5,4 & 15 & 9,1 & 8 & 4,1 \\
\hline Province de Québec & 211 & & 184 & & 164 & & 196 & \\
\hline
\end{tabular}

Tableau 4-Nombre de décès à la suite d'une lésion professionnelle

Source : Statistiques annuelles des années 2012, 2013, 2014 et 2015 de la CSST.

Comme dans tous les milieux professionnels, les travailleurs sont exposés à un certain nombre de risques dont certains ont causé ou peuvent causer des lésions professionnelles. Selon le MSSS (2012), au nombre des contraintes auxquelles les travailleurs de la région sont exposés figurent notamment celles liées à la biomécanique du travail, au bruit, aux exigences psychologiques élevées et aux efforts de travail insuffisamment appréciés (MSSS, 2012).

En effet, le taux d'exposition aux contraintes biomécaniques du travail est plus élevé au sein de la région qu'à l'échelle de la province. Ainsi, $29 \%$ des travailleurs de la région occupent un emploi comportant un niveau élevé de contraintes physiques contre $22 \%$, en moyenne, pour l'ensemble de la province (CIUSSS,
2016). Cette situation d'exposition à des contraintes physiques doublée d'une importance relative des blessures causées par des mouvements répétitifs favorisent l'apparition de lésions liées aux troubles musculosquelettiques (TMS) : $26 \%$ des travailleurs de la région sont victimes de TMS avec un impact sur leurs activités quotidiennes (CIUSSS, 2016).

La proportion de travailleurs aux prises avec des contraintes sonores (exposition au bruit) est également plus élevée dans la région (12\%) que dans le reste du Québec (7\%) (CIUSSS, 2016). L'exposition à des contraintes sonores peut entraîner la surdité professionnelle. Selon les données compilées par l'INSPQ (2014), 337 cas de surdité professionnelle ont été acceptés et indemnisés par la CSST $^{5}$, en 2010, sur un 
total de 4341 cas à l'échelle de la province. Entre 1997 et 2010, la région a connu 2589 cas de surdité professionnelle indemnisés par la CSST représentant 7,2\% du total enregistré dans la province (INSPQ, 2014). Ce qui place le Saguenay-Lac-Saint-Jean à la sixième place derrière la Montérégie, la Mauricie et le Centredu-Québec, Montréal et Chaudière-Appalaches. Ce qui fait que la surdité professionnelle est plus fréquente dans la région $(5,2 \%)$ que dans le reste du Québec $(3,4 \%)$ selon le CIUSSS du Saguenay-LacSaint-Jean (2016).

En ce qui concerne les risques psychologiques, les travailleurs de la région sont moins exposés à des exigences psychologiques élevées $(31 \%)$ comparativement au reste de la province (35\%) (MSSS, 2012). C'est ainsi que pour une des formes de risques psychologiques, à savoir le harcèlement psychologique au travail, la proportion de la population déclarant ne jamais avoir subi de harcèlement psychologique au travail est plus importante dans la région (83\%) qu'au Québec (79 \%) et celle déclarant avoir été victime de harcèlement psychologique au travail de façon occasionnelle est plus faible dans la région $(15 \%)$ qu'au Québec (17\%) (CIUSSS 2016). Cette situation se reflète dans le nombre relativement bas de lésions attribuables au stress et à la violence en milieu de travail acceptées par la CNESST (tableaux 5 et 6).

Selon la CNESST (2017d), une lésion de stress aigu est une lésion qui résulte d'un événement traumatique (accident grave, vol à main armée, violence physique, menaces de mort, etc.) tandis qu'une lésion de stress chronique est une lésion qui découle d'un stress qui se poursuit sur une longue période, par exemple, le harcèlement au travail, le stress lié à la tâche et à l'organisation du travail, etc.

De 2012 à 2015, les lésions professionnelles attribuables au stress en milieu de travail acceptées par la CNESST au profit de travailleurs de la région du Saguenay-Lac-Saint-Jean sont, cumulativement, au nombre de 121 contre 4057 pour l'ensemble du Québec (tableau 5).

\begin{tabular}{|r|r|c|c|c|c|c|c|c|}
\hline & \multicolumn{2}{|c|}{2012} & \multicolumn{2}{c|}{2013} & \multicolumn{2}{c|}{2014} & \multicolumn{2}{c|}{2015} \\
\cline { 2 - 9 } & Nombre & $\%$ & Nombre & $\%$ & Nombre & $\%$ & Nombre & $\%$ \\
\hline $\begin{array}{l}\text { Nombre de lésions attribuables au stress aigu en } \\
\text { milieu de travail }\end{array}$ & & & & & & & & \\
\hline Saguenay-Lac-St-Jean & 27 & 2,9 & 23 & 2,6 & 33 & 3,9 & 22 & 2,4 \\
\hline Province de Québec & 919 & & 883 & & 848 & & 919 & \\
\hline $\begin{array}{l}\text { Nombre de lésions attribuables au stress } \\
\text { chronique en milieu de travail }\end{array}$ & & & & & & & & \\
\hline Saguenay-Lac-St-Jean & 7 & 5,9 & 2 & 1,7 & 4 & 3,1 & 3 & 2,7 \\
\hline Province de Québec & 119 & & 116 & & 131 & & 112 & \\
\hline $\begin{array}{l}\text { Nombre total de lésions attribuables au stress en } \\
\text { milieu de travail }\end{array}$ & & & & & & & & \\
\hline Saguenay-Lac-St-Jean & 34 & 3,3 & 25 & 2,5 & 37 & 3,8 & 25 & 2,4 \\
\hline Province de Québec & 1042 & & 1000 & & 983 & & 1032 & \\
\hline
\end{tabular}

Tableau 5 - Nombre de lésions attribuables au stress en milieu de travail acceptées par la CNESST Source : CNESST (2017e). Statistiques sur les lésions attribuables au stress en milieu de travail, 2012-2015.

Les cas de harcèlement psychologique acceptés et indemnisés par la CNESST, de 2012 à 2015, dans ce lot de lésions attribuables au stress en milieu de travail, sont limités étant donné que sur les 4057 dossiers acceptés à l'échelle provinciale, seuls 152 cas de harcèlement psychologique sont notés. Ce nombre limité de cas de harcèlement psychologique est parcellaire et ne rend pas compte du phénomène dans sa totalité. En effet, pour une seule année (2014-2015), la Commission des normes du travail (CNT) fait état de
3092 plaintes pour harcèlement psychologique déposées en vertu de la Loi sur les normes du travail, L.R.Q., c. N-1.1 (LNT) pour l'ensemble de la province et 60 plaintes pour la seule région du Saguenay-LacSaint-Jean (CNT, 2017).

Le nombre cumulé de dossiers pour lésions psychiques et physiques attribuables à la violence en milieu de travail est de 251 sur un total provincial de 7723 cas en 4 ans (tableau 6). 


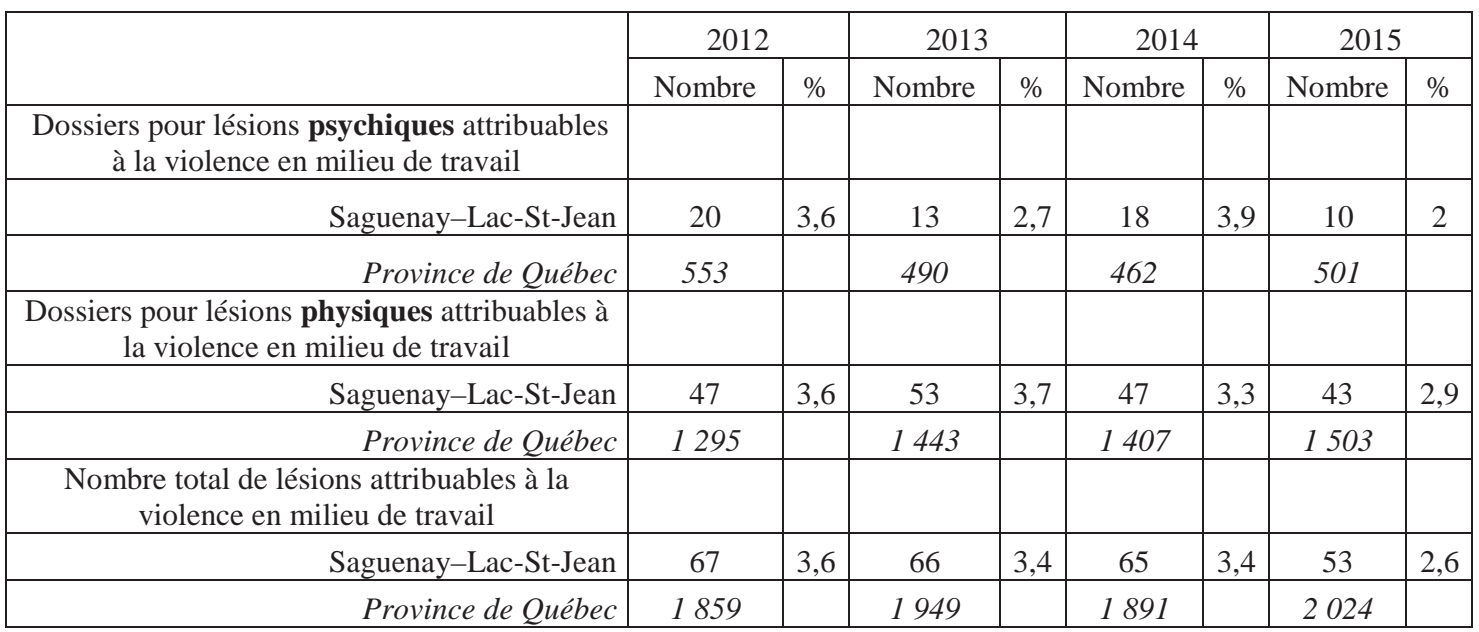

Tableau 6- Lésions attribuables à la violence en milieu de travail acceptées par la CNESST

Source : CNESST (2017e). Statistiques sur les lésions attribuables à la violence en milieu de travail, 2012-2015.

\subsection{Portrait des initiatives de prévention dans la région}

Pour réaliser ses objectifs principaux, c'est-à-dire l'élimination des dangers à la source et la participation des travailleurs, la Loi sur la santé et la sécurité $d u$ travail, L.R.Q., c. S-2.1 (LSST) prévoit quatre mécanismes de gestion de la prévention au sein des entreprises : le programme de prévention (PP), le programme de santé spécifique à l'établissement (PSSE), le comité de santé et de sécurité (CSS) et le représentant à la prévention (RP).

\section{Programme de prévention (PP)}

Le programme de prévention est un plan d'action en prévention propre à chaque établissement, élaboré par l'employeur avec la participation des travailleurs, qui vise à éliminer ou à contrôler les dangers au travail et comporte des mesures concrètes pour y arriver (CNESST, 2017b). Il est obligatoire pour toutes les entreprises relevant des groupes prioritaires 1, 2 et 3, soit l'ensemble des entreprises des SAE de 1 à 15 (tableau 1). Le programme de prévention est également obligatoire pour toutes les entreprises membres d'une mutuelle de prévention. Aucune statistique renseignant sur le nombre de programmes de prévention élaborés régionalement n'a été trouvée. La seule information retrouvée à ce sujet est ancienne et concerne toute la province. Elle est relative au taux de conformité des entreprises québécoises, des groupes prioritaires 1, 2 et 3, ayant l'obligation d'élaborer puis de transmettre à la CNESST un programme de prévention (établissements comptant plus de 20 travailleurs et chantiers de construction devant occuper simultanément au moins 25 travailleurs). Selon Plasse (1988), citée par Baril-Gingras, Vézina et Lippel (2013), $86 \%$ des établissements de plus de 20 travailleurs concernés avaient rempli cette obligation en 1985.

Aucune information n'est, non plus, disponible relativement au nombre de programmes de prévention élaborés par les mutuelles de prévention qui opèrent dans la région. Selon la CSST (2009), une mutuelle de prévention est un regroupement d'employeurs qui s'engage dans une démarche de prévention, de réadaptation et de retour en emploi des travailleurs victimes d'une lésion professionnelle et qui, en contrepartie, bénéficie d'une tarification qui reflète ses efforts en faveur de la santé et de la sécurité. La CNESST (2017c) dénombre 71 mutuelles de prévention qui opèrent dans la région du Saguenay-Lac-St-Jean. Une seule parmi elles est composée uniquement d'employeurs de la région. En dehors d'elle, toutes les autres mutuelles regroupent des employeurs issus d'au moins deux à plusieurs régions de la province.

\section{Programme de santé spécifique à l'établissement (PSSE)}

La LSST exige que tout programme de prévention contienne un programme de santé spécifique à l'établissement (PSSE). Comme tout programme en santé, le PSSE est constitué par un ensemble de ressources réunies et mises en œuvre pour fournir aux travailleurs et aux employeurs des services organisés de façon cohérente dans le temps et dans 
l'espace en vue d'atteindre des objectifs déterminés en rapport avec un problème de santé précis (Drouin et al., 2004). Son élaboration, qui relève du médecin responsable des services de santé de l'établissement, doit se faire en concertation avec le milieu de travail tout en tenant compte des priorités régionales et provinciales en matière de SST. En 2015, le nombre de PSSE élaborés par l'équipe régionale du Réseau de santé publique en santé au travail s'établit à 140 (RSAP, 2017). Ce chiffre paraît plus que modeste en comparaison du nombre d'entreprises des groupes prioritaires 1, 2 et 3 implantées dans la région, mais tout à fait justifié au regard des effectifs restreints de l'équipe régionale de santé publique dédiée à la santé au travail.

\section{Comité de santé et de sécurité et représentant à la prévention}

Le manque d'informations statistiques exhaustives et fiables se révèle aussi en ce qui concerne les comités de santé et de sécurité du travail (CSS) et le représentant à la prévention (RP), autant au niveau provincial que régional. Le comité de santé et de sécurité du travail est un organe interne de prévention à composition paritaire (employeur - travailleurs). Il est rendu obligatoire dans tous les établissements des groupes prioritaires 1 et 2 comptant plus de 20 travailleurs. Quant aux représentants à la prévention, ce sont des travailleurs désignés au sein des entreprises où un comité de santé et de sécurité peut être formé.

L'une des mesures phares de prévention découlant de la LSST est le Programme pour une maternité sans danger (PMSD). Le PMSD a pour objectif principal de maintenir en emploi la travailleuse enceinte ou qui allaite en lui permettant de réaliser des tâches qu'elle est raisonnablement en mesure d'accomplir sans compromettre sa santé ou celle de son enfant. Entre 2012 et 2015, au titre de ce programme, 5442 dossiers de réclamation ont été acceptés au profit de travailleuses de la région, soit $4 \%$ en moyenne, du nombre total enregistré au niveau provincial au cours de la même période (tableau 7).

\begin{tabular}{|r|c|c|c|c|c|c|c|c|}
\hline \multirow{2}{*}{} & \multicolumn{2}{|c|}{2012} & \multicolumn{2}{c|}{2013} & \multicolumn{2}{c|}{2014} & \multicolumn{2}{c|}{2015} \\
\cline { 2 - 9 } & Nombre & $\%$ & Nombre & $\%$ & Nombre & $\%$ & Nombre & $\%$ \\
\hline $\begin{array}{r}\text { Nombre de réclamations de } \\
\text { travailleuses enceintes au PMSD }\end{array}$ & & & & & & & & \\
\hline Saguenay-Lac-St-Jean & 1358 & 4,1 & 1360 & 4,1 & 1369 & 4,2 & 1328 & 4 \\
\hline Province de Québec & 33100 & & 32921 & & 32835 & & 32799 & \\
\hline $\begin{array}{l}\text { Nombre de réclamations de } \\
\text { travailleuses qui allaitent au PMSD }\end{array}$ & & & & & & & & \\
\hline Saguenay-Lac-St-Jean & 10 & 4 & 5 & 1,9 & 8 & 3,1 & 4 & 1,5 \\
\hline Province de Québec & 249 & & 266 & & 262 & & 259 & \\
\hline $\begin{array}{r}\text { Nombre total de réclamations faites au } \\
\text { titre du PMSD }\end{array}$ & & & & & & & & \\
\hline Saguenay-Lac-St-Jean & 1368 & 4,1 & 1365 & 4,1 & 1377 & 4,2 & 1332 & 4 \\
\hline Province de Québec & 33349 & & 33187 & & 33097 & & 33058 & \\
\hline
\end{tabular}

Tableau 7 - Réclamations concernant le programme pour une maternité sans danger (PMSD) acceptées par la CNESST Source : CNESST (2017). Pour une maternité sans danger : Statistiques 2012-2015.

En ce qui concerne les interventions préventives réalisées par la CNESST au sein de certaines entreprises en activité dans le ressort territorial de la région du Saguenay-Lac-St-Jean, on constate une tendance à la baisse de leur nombre : de 1058 dossiers d'intervention en 2013, on est passé à 879 en 2015 (tableau 8). De même, le nombre de visites effectuées par les inspecteurs de la CNESST en lien avec ces dossiers d'intervention connaît une réduction sensible en passant de 1959 visites en 2013 à 1650 visites en 2015 (tableau 8). Par ailleurs, les visites inhérentes à l'ouverture de ces dossiers d'intervention ont permis aux inspecteurs de la CNESST de relever des manquements aux obligations prescrites par la LSST en matière de prévention, lesquels ont donné lieu à des suites légales allant des dérogations constatées aux constats d'infraction en passant par les décisions (tableau 8). Ainsi, en moyenne, chaque dossier d'intervention ouvert a permis de constater plus de trois situations dérogatoires à la loi ou aux règlements en SST. 


\begin{tabular}{|c|c|c|c|c|c|c|c|c|}
\hline & \multicolumn{2}{|c|}{2012} & \multicolumn{2}{|c|}{2013} & \multicolumn{2}{|c|}{2014} & \multicolumn{2}{|c|}{2015} \\
\hline & Nombre & $\%$ & Nombre & $\%$ & Nombre & $\%$ & Nombre & $\%$ \\
\hline \multicolumn{9}{|l|}{ Établissements actifs } \\
\hline Saguenay-Lac-St-Jean & 11054 & 4,2 & 11151 & 4,1 & 11214 & 4,1 & 11202 & 4,1 \\
\hline Province de Québec & 265187 & & 269303 & & 270231 & & 272315 & \\
\hline \multicolumn{9}{|l|}{ Dossiers d'intervention en prévention - inspection } \\
\hline Saguenay-Lac-St-Jean & 987 & 6,1 & 1058 & 6,2 & 1068 & 6,2 & 879 & 5 \\
\hline Province de Québec & 16116 & & 17118 & & 17338 & & 17415 & \\
\hline \multicolumn{9}{|l|}{ Visites effectuées } \\
\hline Saguenay-Lac-St-Jean & 1637 & 4,8 & 1959 & 5,5 & 1978 & 5,5 & 1650 & 4,8 \\
\hline Province de Québec & 33791 & & 35907 & & 35655 & & 34515 & \\
\hline \multicolumn{9}{|l|}{ Dérogations constatées } \\
\hline Saguenay-Lac-St-Jean & 3270 & 4,9 & 3843 & 5,4 & 3662 & 5 & 3180 & 4,6 \\
\hline Province de Québec & 66687 & & 71786 & & 72598 & & 68809 & \\
\hline \multicolumn{9}{|l|}{ Décisions prises lors des interventions } \\
\hline Saguenay-Lac-St-Jean & 325 & 6,7 & 268 & 5,4 & 252 & 5,4 & 212 & 5,3 \\
\hline Province de Québec & 4887 & & 4926 & & 4674 & & 3972 & \\
\hline \multicolumn{9}{|l|}{ Constats d'infraction signifiés } \\
\hline Saguenay-Lac-St-Jean & 195 & 4,3 & 278 & 6 & 168 & 3,6 & 135 & 3,6 \\
\hline Province du Québec & 4583 & & 4669 & & 4611 & & 3713 & \\
\hline
\end{tabular}

Tableau 8 - Interventions réalisées par la CNESST et leurs suites légales Source : Statistiques annuelles des années 2012, 2013, 2014 et 2015 de la CSST.

Ces résultats sont à un cran supérieur en matière d'interventions réalisées par la CNESST et des suites légales qui en ont résulté comparativement à la région administrative de l'Estrie qui compte sensiblement le même nombre d'établissements actifs (11 444) qu'au Saguenay-Lac-Saint-Jean (11 202) (CSST, 2016).

\section{Discussion}

La première phase de la recherche s'est effectuée en recueillant et en analysant des données secondaires. $\mathrm{Si}$ cette méthode présente de nombreux avantages (mise en œuvre de ressources limitées, accès rapide aux données, etc.), elle comporte des inconvénients susceptibles de limiter la portée des résultats obtenus. En effet, l'utilisation de données secondaires peut être limitée par l'écart entre les objectifs à la base de la collecte primaire et les objectifs de l'analyse secondaire. Selon Bernatchez et Turgeon (2016, p 509), «les données primaires n'ont pas été mises en forme en tenant compte des objectifs de l'analyse secondaire ». Un autre inconvénient, et non le moindre, est relatif à la non-disponibilité, dans certains cas, des informations désirées. L'exemple du nombre de programmes de prévention élaborés et mis en œuvre au sein des entreprises de la région ou celui du nombre de comités de santé et sécurité du travail fonctionnels en constituent une parfaite illustration. De plus, comme le soulignent Baril-Gingras, Vézina et Lippel (2013), le fait, par exemple, d'élaborer et de transmettre un programme de prévention à la CNESST ne signifie pas que celui-ci est effectivement implanté et de bonne qualité.

S'agissant des résultats, tout indique que la totalité des travailleurs de la région ne sont pas couverts par le régime de santé et sécurité au du travail mis en place et géré par la CNESST si l'on considère que le taux de couverture de celui-ci est d'environ $93 \%$ selon l'INSPQ (2010) sur la base des statistiques de l'Association des commissions des accidents du travail du Canada (ACATC). Par conséquent, les statistiques sur les lésions professionnelles présentées ici ne reflètent pas toute la réalité. En effet, comme le souligne DaubasLetourneux (2008), toutes les maladies potentiellement indemnisables, par exemple, ne donnent pas lieu à une déclaration ou à une reconnaissance, car 
la liste des maladies reconnues comme étant d'origine professionnelle résulte davantage de négociations et de consensus entre les partenaires sociaux que de l'état des connaissances sur les liens entre le travail et les atteintes à la santé. C'est la même chose concernant les accidents du travail qui, selon Daubas-Letourneux (2009), échappent à toute visibilité, car certains ne sont pas déclarés et d'autres le sont, mais ne sont pas reconnus.

En l'absence de données spécifiques à la région, une référence aux statistiques à l'échelle provinciale permet de constater que, de 2012 à 2015, 39 \%, en moyenne, des lésions professionnelles survenues et indemnisées au Québec ont été enregistrées dans les entreprises relevant des groupes prioritaires 1, 2 et 3 contre $54 \%$, en moyenne, pour les groupes prioritaires 4,5 et 6 censés être moins dangereux. Tout porte à croire que les données du SaguenayLac-Saint-Jean semblent épouser les mêmes contours que celles obtenues à l'échelle provinciale quand on sait que 95,2\% des entreprises que compte la région ont moins de 50 travailleurs. Cela met en lumière une évidence déjà soulignée par Champoux et Brun (2003), à savoir que c'est dans les plus petites entreprises, celles qui comptent moins de 50 travailleurs, que surviennent, plus fréquemment, les accidents du travail et les maladies professionnelles. La faiblesse de la prise en charge des questions de SST dans ces entreprises de petite taille s'explique, en partie, par le manque de ressources ainsi que par le cumul de fonctions et la surcharge de travail de leurs principaux responsables (Champoux et Brun, 2000).

\section{Conclusion}

Au-delà des limites inhérentes au recueil et à l'analyse des données secondaires, le portrait esquissé est utile à maints égards en ce sens qu'il permet de disposer d'une vision globale sur la situation régionale en SST notamment sur le nombre de lésions professionnelles qui y sont survenues, les principaux risques auxquels les travailleurs sont exposés, les initiatives de prévention menées au niveau local et, surtout, sur la taille des entreprises implantées dans la région. Ce portrait sommaire comporte toutefois quelques facettes à approfondir du fait de l'inexistence de certaines statistiques qui auraient pu élargir la vision de la situation globale (pas de données, par exemple, sur le nombre de programmes de prévention, de comités de santé et de sécurité du travail, de représentants à la prévention). Toutefois, les résultats obtenus sont suffisamment représentatifs de la situation et, à ce titre, préparent et facilitent la réalisation de la seconde phase. Ils jettent, déjà, un éclairage sur la nécessité d'orienter les recherches et actions futures en tenant compte des particularités et des besoins des entreprises de très petite taille, lesquelles constituent l'essentiel du tissu économique de la région.

\section{NOTES}

1 MSSS : Ministère de la Santé et des Services sociaux CNESST : Commission des normes, de l'équité, de la santé et de la sécurité du travail INSPQ : Institut national de santé publique du Québec ISQ : Institut de la statistique du Québec

2 CLD : Centre local de développement CIUSSS : Centre intégré universitaire de santé et de services sociaux

3 Établissements qui ont été ouverts pendant toute ou partie de l'année.

4 Dossiers ouverts entre le $1^{\text {er }}$ janvier et le 31 décembre pour chaque année visée.

5 La CSST est devenue, depuis le 1er janvier 2016, la Commission des normes, de l'équité, de la santé et de la sécurité du travail (CNESST).

\section{RÉFÉRENCES}

Bernatchez J. et Turgeon, J. (2016). Les données secondaires. Dans B. Gauthier et I. Bourgeois (dir.), Recherche sociale (6e édition). Québec, Québec : Presses de l'Université du Québec.

Centre local de développement (CLD) Maria-Chapdelaine (2017). Répertoire de toutes les entreprises de la région du Saguenay-LacSaint-Jean. Repéré à http://www.maximisation.net/upload/PDF1/812b4ba287f5ee0bc9d43bbf5bbe87fb.pdf. 
Champoux D. et Brun, J.-P. (2000). Prise en charge de la sécurité dans les petites entreprises manufacturières : état de la situation et pistes pour l'intervention et la recherche. Perspectives interdisciplinaires sur le travail et la santé. Repéré à http://pistes.revues.org/3812 DOI : 10.4000/pistes.3812.

Champoux D. et Brun, J.-P. (2003). Occupational health and safety management in small-size enterprises: An overview of the situation and avenues for intervention and research. Safety Science, 41, 301-318.

CIUSSS (2016). Enquête québécoise sur la santé de la population 2014-2015 - Faits saillants pour la région du Saguenay-Lac-Saint-Jean. Centre intégré universitaire de santé et de services sociaux du Saguenay-Lac-St-Jean.

Cloutier-Villeneuve, L. et Demers, M.-A. (2014). Regard statistique sur la converture syndicale au Québec, ailleurs au Canada et dans les pays de l'OCDE. Québec, Québec : Institut de la statistique du Québec.

CNESST (2017). Pour une maternité sans danger: Statistiques 2012-2015. Direction de la comptabilité et de la gestion del'information. Commission des normes, de l'équité, de la santé et de la sécurité du travail du Québec. DC300-254-10 (2017-03).

CNESST (2017a). Liste des employeurs classifiés par groupe prioritaire (1 à 6) pour la région de Saguenay — Lac-Saint-Jean. Unité dédiée, accès à l'information, Commission des normes, de l'équité, de la santé et de la sécurité du travail du Québec. Réponse à la demande d'accès 2017 N/D : 146266DAJ. Repéré à http://www.cnesst.gouv.qc.ca/acces-information/diffusion-de-l-information/Documents/146266DAJ-1.pdf.

CNESST (2017b). Programme de prévention. Repéré à http://www.csst.qc.ca/prevention/programme_prevention/pages/programme_prevention.aspx.

CNESST (2017c). Listes des Mutuelles de prévention. Sélection par critères de recherche. Commission des normes, de l'équité, de la santé et de la sécurité du travail du Québec. Outil de sélection en ligne. Repéré à http://www.csst.qc.ca/asp/ListeDesMutuelles/mutuelle.asp.

CNESST (2017d). Statistiques sur les lésions attribuables au stress en milieu de travail, 2012-2015. Centre de la statistique et de l'information de gestion, Direction de la comptabilité et de la gestion de l'information. Commission des normes, de l'équité, de la santé et de la sécurité du travail du Québec. DC300-321-7 (2017-03).

CNESST (2017e). Statistiques sur les lésions attribuables à la violence en milieu de travail, 2012-2015. Centre de la statistique et de l'information de gestion, Direction de la comptabilité et de la gestion de l'information. Commission des normes, de l'équité, de la santé et de la sécurité du travail du Québec. DC300-255-9 (2017-03).

CNT (2017). Données sur le traitement des plaintes déposées en vertu de la Loi sur les normes du travail. Répartition géographique des plaintes reçues, 2014-2015. Commission des normes du travail. Tableau en ligne. Repéré à http://www.cnt.gouv.qc.ca/filead$\mathrm{min} / \mathrm{pdf} /$ publications/Re_partition_ge_ographique_des_plaintes_rec_ues_2014-2015_.pdf.

CSST (2009). Mutuelles de prévention. Ce que vous devez savoir. Commission de la santé et de la sécurité du travail du Québec, DC200-1434-3 (2010-04). ISBN : 978-2-550-55072-3.

CSST (2013). Statistiques annuelles de 2012. Direction de la comptabilité et de la gestion de l'information, Commission de la santé et de la sécurité du travail du Québec. DC200-1046-20 (2013-10). ISBN : 978-2-550-68821-1 (PDF).

CSST (2014). Statistiques annuelles de 2013. Direction de la comptabilité et de la gestion de l'information, Commission de la santé et de la sécurité du travail du Québec. DC200-1046-21 (2014-11). ISBN : 978-2-550-71286-2 (PDF).

CSST (2015). Statistiques annuelles de 2014. Direction de la comptabilité et de la gestion de l'information, Commission de la santé et de la sécurité du travail du Québec. DC200-1046-22 (2015-10). ISBN : 978-2-550-73874-9 (PDF).

CSST (2016). Statistiques annuelles de 2015. Direction de la comptabilité et de la gestion de l'information, Commission de la santé et de la sécurité du travail du Québec. DC200-1046-23 (2016-10). ISBN : 978-2-550-76935-4 (PDF).

Daubas-Letourneux, V. (2008). Produire des connaissances en santé au travail à l'échelle régionale. Le signalement des maladies à caractère professionnel dans les Pays de la Loire. Revue française des affaires sociales, 2008/2, 213-235.

Daubas-Letourneux, V. (2009). Accidents du travail : des blessés et des morts invisibles. Mouvements, 2009/2 (58), 29-37. DOI $10.3917 /$ mouv.058.0029.

Drouin L., Simard, R., Fontaine, G. et Huneault, C. (2004). L'élaboration et la mise en application des programmes de santé spécifiques aux établissements : l'approche québécoise. Santé publique, 2004/2 (16), 200. DOI : 10.3917/spub.042.0329.

Fortin M.-F. et Gagnon, J. (2016). Fondements et étapes du processus de recherche : méthodes quantitatives et qualitatives (3e éd.). Montréal, Québec : Chenelière Éducation. 
Baril-Gingras, G., Vézina, M. et Lippel, K. (2013). Bilan relatif aux dispositions de la LSST : Vers une application intégrale? Relations industrielles, 684(2013), 682-708. DOI : 10.7202/1023011ar.

INSPQ (2010). Portrait national des troubles musculosquelettiques (TMS) 1998-2007. TMS sous surveillance. Institut national de santé publique du Québec. ISBN : 978-2-550-59984-5 (PDF).

INSPQ (2014). Portrait de la surdité professionnelle acceptée par la Commission de la santé et de la sécurité du travail au Québec: 1997-2010. Troubles de l'audition sous surveillance. Direction des risques biologiques et de la santé au travail, Institut national de santé publique du Québec. ISBN : 978-2-550-69879-1 (PDF).

ISQ (2017a). Panorama des régions du Québec. Édition 2017. Institut de la statistique du Québec. ISBN : 978-2-550-76006-1 (PDF).

ISQ (2017b). Résultats de l'Enquête sur la population active pour le Québec. Données désaisonnalisées de juillet 2017. Direction des statistiques du travail et de la rémunération. Institut de la statistique du Québec. ISBN : 1927-2251 (PDF).

Mayer, R. (2000). L'analyse des milieux et des problèmes sociaux. Dans R. Mayer, F. Ouellet, M.-C. Saint-Jacques et D. Turcotte (dir.). Méthodes de recherche en intervention sociale (p. 233-255). Montréal, Québec : Édition Gaëtan Morin.

MSSS (2012). Le travail : un déterminant important de la santé. Direction générale de la santé publique, ministère de la Santé et des Services sociaux, ISBN : 978-2-550-64173-5 (PDF).

Plasse, M. (1988). Santé et sécurité du travail : De la confrontation à la concertation. Montréal, Québec : Éditions Agence d'ARC inc.

RSAP (2017). La région en quelques chiffres. Réseau de santé publique en santé au travail. Saguenay-Lac-Saint-Jean. Repéré à http://www.santeautravail.qc.ca/web/region-saguenay-lac-saint-jean/accueil. 